\title{
Innovative DC Commutator-less Drive
}

Author: Bahram Amin. Ph.D. Formerly Senior Researcher at the Research National Institute of Transportation (INRETS), France; Presently in retirement (since 2011). Not registered in ORCID. Tel: (+33) 621699610Email: bahramyn75@outlook.com

\begin{abstract}
In this paper we shall present a new category of DC electrical drive called "DC commutator-less drives". The control technic of these category of drives is in all aspect similar to that of a classical DC Commutator Motor. It consists in regulating the level of its input voltage using a DC/DC converter (a Chopper) or a AC/DC converter (phase-controlled rectifier bridge); the motor, however, does not have a mechanical commutator and do not use sliding brushes. In other words, the drive has all the advantages of a classical DC drive for variable speed applications without the drawbacks.
\end{abstract}

\section{1- Introduction}

With the apparition of industrial solid power thyristors in the early 1970's, utilization of the classical DC Commutator Motor for variable speed applications in various industrial branches became popular. In fact, it became easy to fabricate simple and efficient DC/DC or AC/DC converters using power thyristors. Such converters, commonly called "choppers" or "phasecontrolled rectifier bridges", easily transform the constant AC voltage or DC voltage of a source at its input into adjustable voltages at its output. The output voltage is applied using a mechanical commutator and sliding brushes to the armature winding of a DC motor for feeding and also controlling the motor: The higher the output voltage of the chopper, the faster the motor shaft revolves. Such a drive (DC commutator motor + appropriate converter) is referred to as a chopper-controlled DC commutator drive or a phase-controlled rectifier DC commutator drive. For illustration purposes, FIG.1 represents the schematic of a choppercontrolled DC commutator drive. In this figure the DC source of constant voltage is represented by a battery the positive terminal of which is connected to a L-C filter. The output of the filter capacitor $\mathrm{C}$ is connected the input of a step-down chopper formed by a controllable Thyristor $\mathrm{TH}$, and a freewheeling diode $\mathrm{D}$, which, together, constitute the DC/DC converter. The output of the converter is connected using a mechanical commutator and sliding brushes to the armature windings of a DC motor. Such simple drives have been used (and, in many places, are still used) in variable speed drive applications, including for powering locomotives in railways transportation.

Despite its very practical and effective control circuitry using a simple DC/DC converter (chopper), this category of drives exhibits the drawback of using classical DC motors with mechanical commutators and sliding brushes which are expensive to fabricate, subject to wear and necessitate frequent maintenance. Furthermore, they constitute an obstacle to high speed functioning of the motor, limiting the power density (power per unit weight) of the drive.

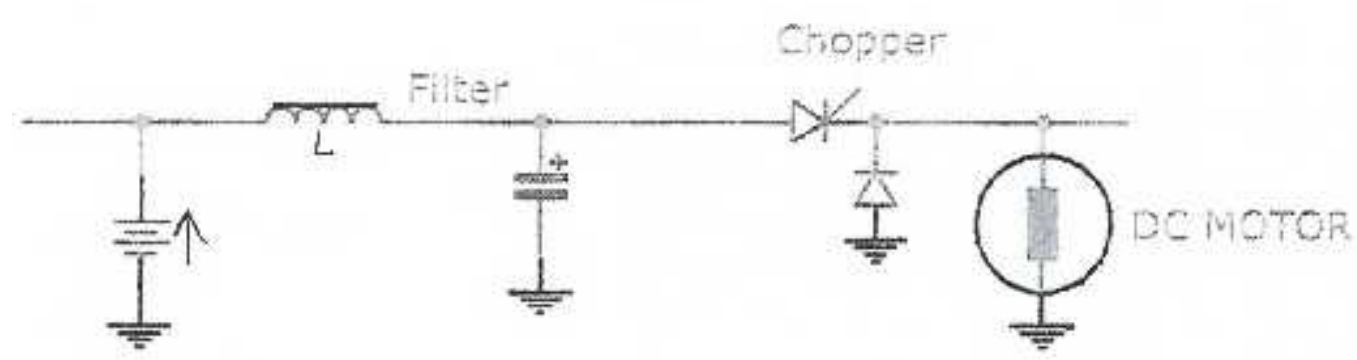

FIG.1-

Chopper-Controlled DC Commutator Motor 
This is why revolving field, inverter-fed AC drives using AC motors, which do not require mechanical commutators, are nowadays becoming more popular than the chopper controlled DC commutator motors. In the following sections, we present a new category of DC motors without mechanical commutators and sliding brushes, namely DC commutator-less motors. DC commutator-less motors can also be fed and controlled through a DC/DC converter (chopper) and constitute a new category of drives naturally called "Chopper controlled DC commutator-less drives." This is genuinely a new category of drives, not known and not mentioned in the technical literature.

\section{2- Commutator-less DC motor}

As stated above, by definition, a DC motor is the one which can be fed and controlled through a DC/DC converter (chopper) or an AC/DC phase-controlled rectifier. The well-known classical DC commutator motors are examples of such a device using a mechanical commutator and sliding brushes. It is possible, however, to design a DC motor, that is, a motor fed and controlled by a DC/DC or AC/DC converter, which has not mechanical commutator and sliding brushes (do not confuse this category of motors with the so called "brushless DC motors" which are actually AC motors). Indeed, in this category of motors currents commute electronically between the different sections (branches) of the armature windings. An example of such a motor is a doubly salient reluctance motor (SRM) functioning in the single-shoot mode. Such a motor is fed and controlled using two voltage sources: One voltage source called $\mathrm{U} 1$, which is adjustable (say from U1min to U1max); and, a second source of constant voltage, U2. It is assumed, however, that U2 is much greater than U1max (say U2=2U1max).

The FIG. 2 shows a 6 -phase bipolar SRM having $\mathrm{Ns}=12$ teeth on the stator an $\mathrm{Nr}=10$ teeth on the rotor. When the position of the rotor is such that the reluctance of a phase Phk is maximum, we say that the rotor is in the electrical angle position of $0^{\circ}$ with respect to the phase Phk. When the position of the rotor is such that the reluctance of a phase $\mathbf{P h}$ is minimum, we say that the rotor is in the electrical angle position of $180^{\circ}$ with respect to the phase Phk. The relation between the mechanical angle position " $\Theta$ " and the electrical angle position " $\theta$ " is: $\theta=\mathrm{Nr} . \Theta$.

The motor shown in FIG.2 is currently referred to as a $12 / 10$ motor or M12/10. This combination of number of teeth on the stator and on the rotor is advantageous, since it allows the motor to have at any instant three phases in a torque productive position. These phases can be determined at any instant by providing the rotor with an absolute angular position sensor. The cyclic sequence of three interlaced torque productive phases in a M12/10 and that of the four interlaced torque productive phases in M16/14 are shown below:

Interlaced Phases of M12/10

$\begin{array}{lll}\text { PH1 } & \text { PH2 } & \text { PH3 } \\ \text { PH2 } & \text { PH3 } & \text { PH4 } \\ \text { PH3 } & \text { PH4 } & \text { PH5 } \\ \text { PH4 } & \text { PH5 } & \text { PH6 } \\ \text { PH5 } & \text { PH6 } & \text { PH1 } \\ \text { PH6 } & \text { PH1 } & \text { PH2 } \\ \text { PH1 } & \text { PH2 } & \text { PH3 }\end{array}$

Interlaced Phases of M16/14

$\begin{array}{llll}\text { PH1 } & \text { PH2 } & \text { PH3 } & \text { PH4 } \\ \text { PH2 } & \text { PH3 } & \text { PH4 } & \text { PH5 } \\ \text { PH3 } & \text { PH4 } & \text { PH5 } & \text { PH6 } \\ \text { PH4 } & \text { PH5 } & \text { PH6 } & \text { PH7 } \\ \text { PH5 } & \text { PH6 } & \text { PH7 } & \text { PH8 } \\ \text { PH6 } & \text { PH7 } & \text { PH8 } & \text { PH1 } \\ \text { PH7 } & \text { PH8 } & \text { PH1 } & \text { PH2 } \\ \text { PH8 } & \text { PH1 } & \text { PH2 } & \text { PH3 } \\ \text { Ph1 } & \text { ph2 } & \text { PH3 } & \text { PH4 }\end{array}$




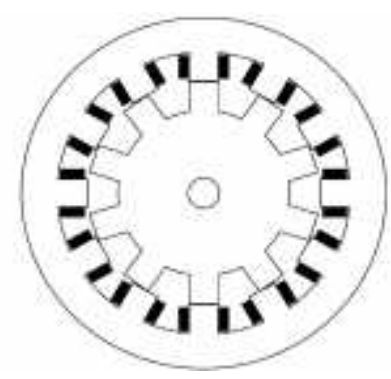

FIG.2- $12 / 10$ MOTOR

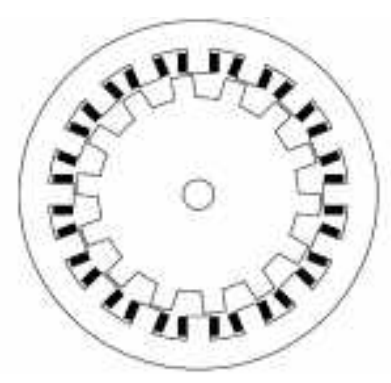

FIG.3- 16/14 MOTOR

Noted that one can consider an SRM with any even number of phases, $\mathrm{Nph}=2 \mathrm{~s}$. If the number of poles per phase is noted " $2 p$ ", the number of teeth on the stator would be Ns=4ps. If we chose the number of the rotor teeth equal to $\mathrm{Nr}=2 p(2 s-1)$, such a motor would have at any instant " $\mathrm{s}$ " phases in torque productive positions. In a single-shoot mode functioning, each phase turning OFF is replaced at the same time by another phase turning ON so that at any instant there are " $\mathrm{s}$ " regularly shifted motoring torque productive phases in action. If the rotor completes one revolution per second, that is, during one cycle, each power switch turns $\mathrm{Nr}$ times ON and Nr times OFF; thus the switching frequency of that phase would be is Nr. Now, If the rotor completes " $n$ " revolutions per minute (RPM), the phase switching frequency in the single-shoot mode functioning would be: $f s=(n / 60) N r=2 p(2 s-1)(n / 60)$. This equality shows that for a given rotor angular speed, the phase switching frequency is minimum for a bipolar phase design, that is, for $p=1$. We say that such a motor has a standard design structure.

Let us assume that the sense of the winding of each pole of a phase is chosen so as when current circulates in this phase one of the pole becomes magnetically positive and the other pole magnetically negative. Under this circumstances all the phases of the machine remain mutually independent. For example, in referring to the 6-phase machine in FIG.2, if a current circulates, say, in phase-1, the magnetic flux linked by the phase- 3 up to the phase- 5 is rigorously nil. In fact, assuming that the motor structure has a perfect center-symmetry, it is easy to show that when an arbitrary current circulates in the Phase-1, the magnetic potential of the stator yoke and that of the rotor yoke are equal. In that case no magnetic flux can leave the rotor yoke to reach the stator yoke. That is, phase- 1 and phase- 3 up to phase- 5 inclusive remain mutually uncoupled. However, the two phases adjacent to phase- 1 , that is, phase- 2 and phase- 6 , can experience a very weak magnetic flux linkage because of the small leakage flux traversing the two slots lodging the winding's arms of the phase-1 and linking with the windings of the phase- 2 and the phase- 6 . This phenomenon can be checked experimentally using a sensitive voltmeter. In this way we can state that the motor shown in FIG.2 represents six independent identical motors space-shifted by an angle $360^{\circ} / 6=60^{\circ}$. We conclude that the variations of their torque as well as their phase currents versus the angular position are identical, except that they are space-shifted by an angle of $60^{\circ}$. If the instant current circulating in the Phase- $k$ is $l_{k}$ and if each phase-k develops the instant torque $T_{k}$, the resultant instant torque developed on the motor's shaft would be:

$$
\mathrm{T}=\sum \mathrm{T}_{\mathrm{k}}
$$

What is stated above for the $12 / 10$ motor of the $\mathbf{F} .2$ remains valid for the $16 / 14$ motor of the FIG.3 and for any other motor structure having a standard design structure.

Now assume that the same pattern of current versus angular positions circulates in the various phases of a 12/10 motor. As explained above, the various curves of torque developed by each phase are identical and can be deduced from each other by a translation along the angular position axis equal to a multiple of $60^{\circ}$. For a $16 / 14$ motor, the translation angle would be a multiple of $360^{\circ} / 8=45^{\circ}$. The particularity of being allowed to consider a $12 / 10$ motor 
working simultaneously as a set of six interlaced single phase motor, each phase being shifted $60^{\circ}$ in respect to its adjacent phase, is very important when one wants to estimate the rate of the torque ripples of the resultant torque developed on the shaft. In fact, assume the pattern of the current in a single phase of the motor to be as follows:

Iph $=\mathrm{I}\left(\right.$ constant for electrical position angle from $0^{\circ}$ to $\left.180^{\circ}\right)$

Iph $=0$ (nil for electrical position angle from $180^{\circ}$ to $360^{\circ}$ )

The shape of the torque's curve versus position angles depend on the level of the phase current, "I" and fact that whether the magnetic circuit becomes saturated or not. In an unsaturated case, the curves of the torque versus the position angles resemble more and less to a sine curve as one can verify it on FIG.4. The resultant torque of the three interlaced phases of a M12/10 and that of the four interlaced phases of a M16/14 are shown in FIG.5 and FIG.6.

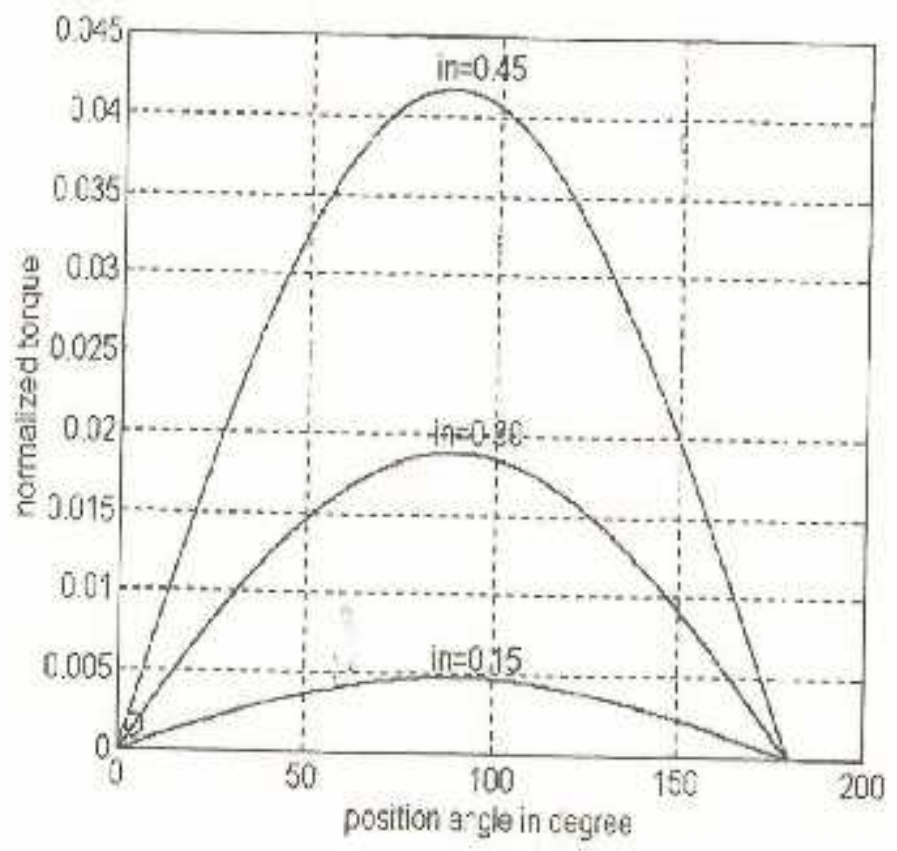

FIG.4- Torque of an individual phase for constant current versus position angles, unsaturated case 


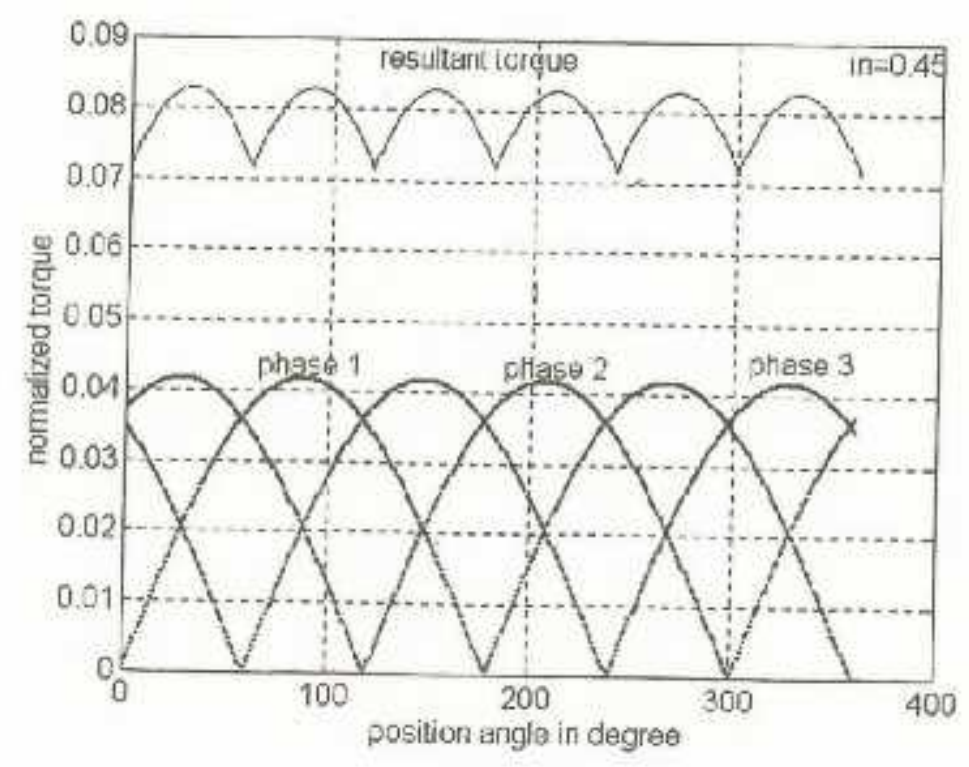

FIG.5- individual torque per phase and resultant torque of 12/10 motor, unsaturated case

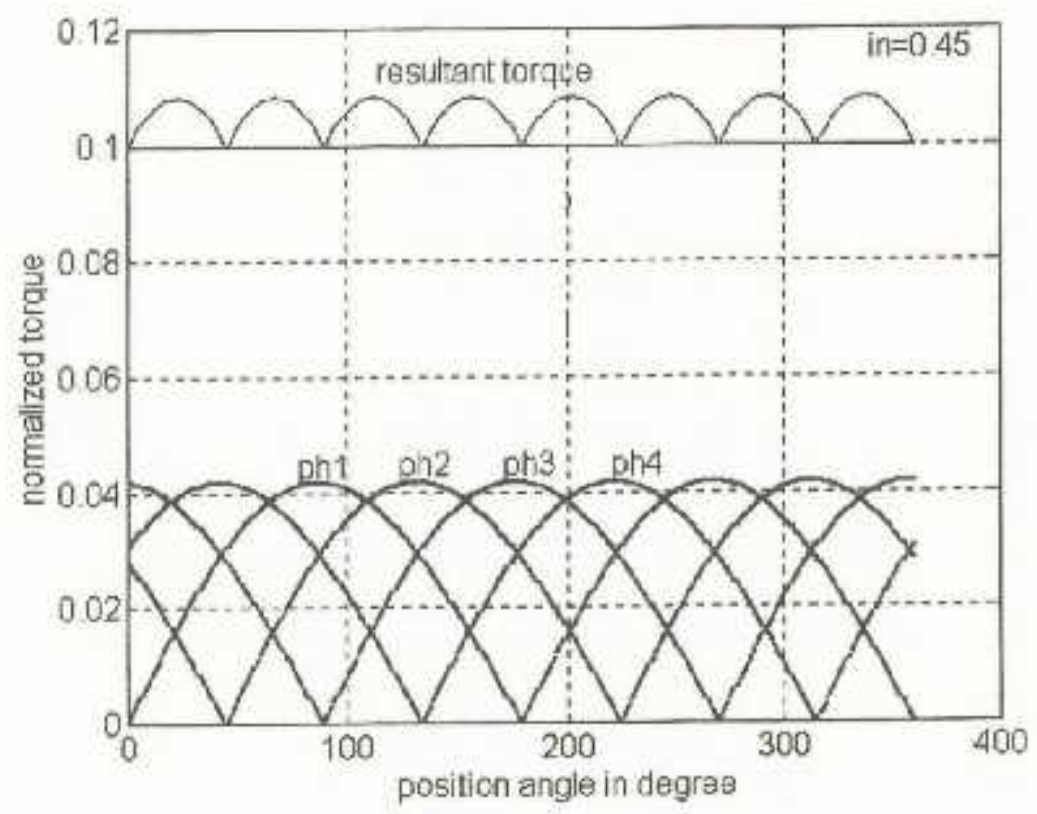

FIG.6- Individual torque per phase and resultant torque of 16/14 motor, unsaturated case

It is clear that torque ripples of the resultant torque are much reduced in comparison to that of each individual phase, as can be verified on FIG.5 and FIG.6. Actually, if we define the rate of the torque ripples of each single phase over the half angular period $180^{\circ}$ by the following relation: 


$$
\mathrm{RT}=\frac{T \quad-T}{T}
$$

It is easy to show that for a torque curve having the shape of a sine wave over the half period, the rate of the torque ripples, given by equation (2), could be analytically calculated as:

$$
\mathrm{RT}_{\mathrm{T}}=\frac{u}{2 \mathrm{~S}}\left[\frac{1}{\mathrm{~S}\left(\frac{n}{2 S}\right)}-\frac{1}{\mathrm{t}_{\mathrm{i}}\left(\frac{n}{2 S}\right)}\right]
$$

In the case where the phase current is high and the magnetic circuit of the machine is saturated, the shape of torque over the half period $180^{\circ}$ is slightly different from a sine curve and is rather as shown in FIG.7. However, the resultant torques are still similar to that of the non-saturated case, as can verified on FIG.8 and FIG.9. Hence, comparing FIG.8 and FIG.9 with FIG.5 and FIG.6 respectively, we conclude that the rate of the torque ripples in a saturated case could be calculated quite accurately by equation (3) as well.

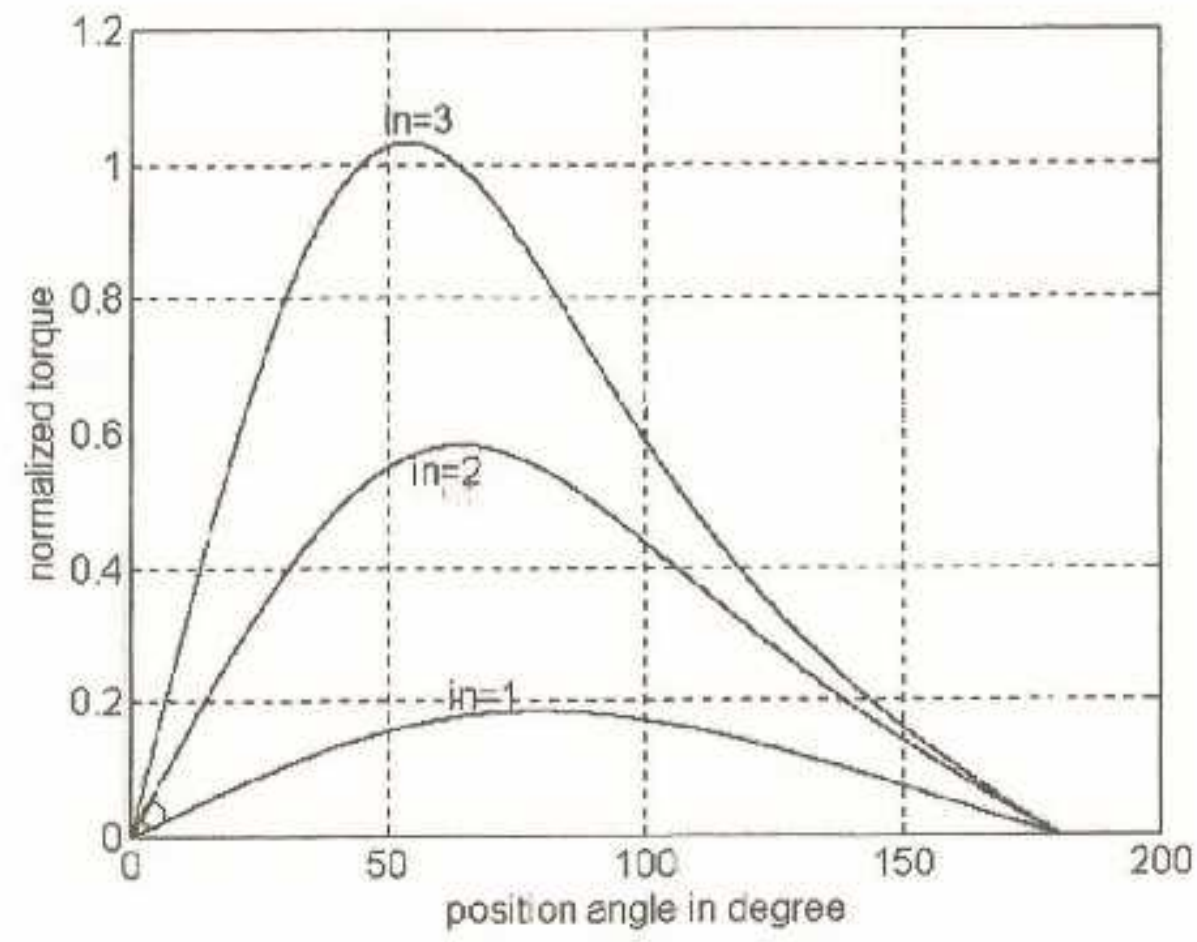

FIG.7- Torque of an individual phase for constant current versus position angles, saturated case 


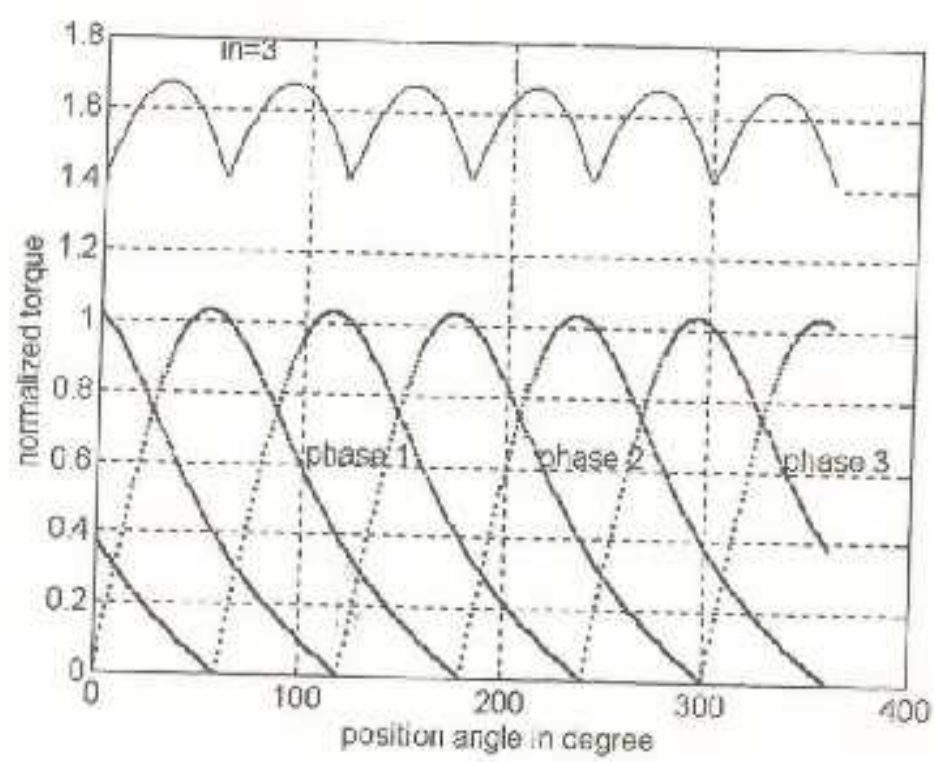

FIG.8- Individual torque per phase and resultant torque of 12/10 motor, saturated case

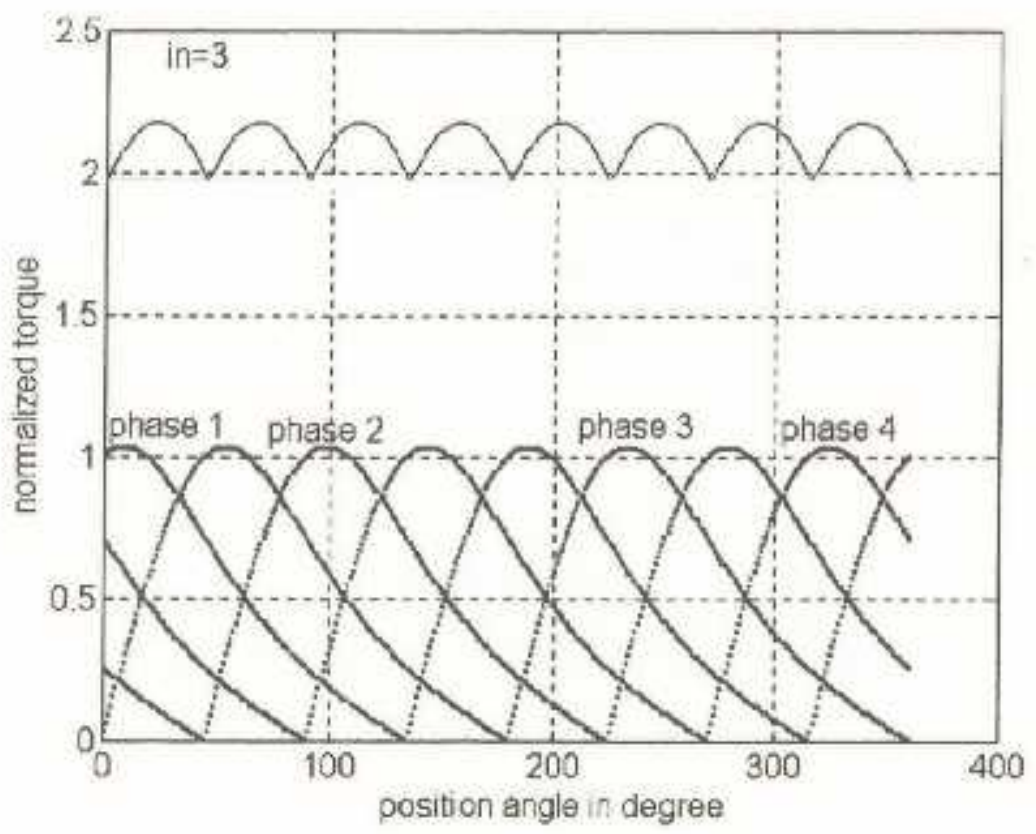

FIG.9- Individual torque per phase and resultant torque of 16/14 motor, saturated case 

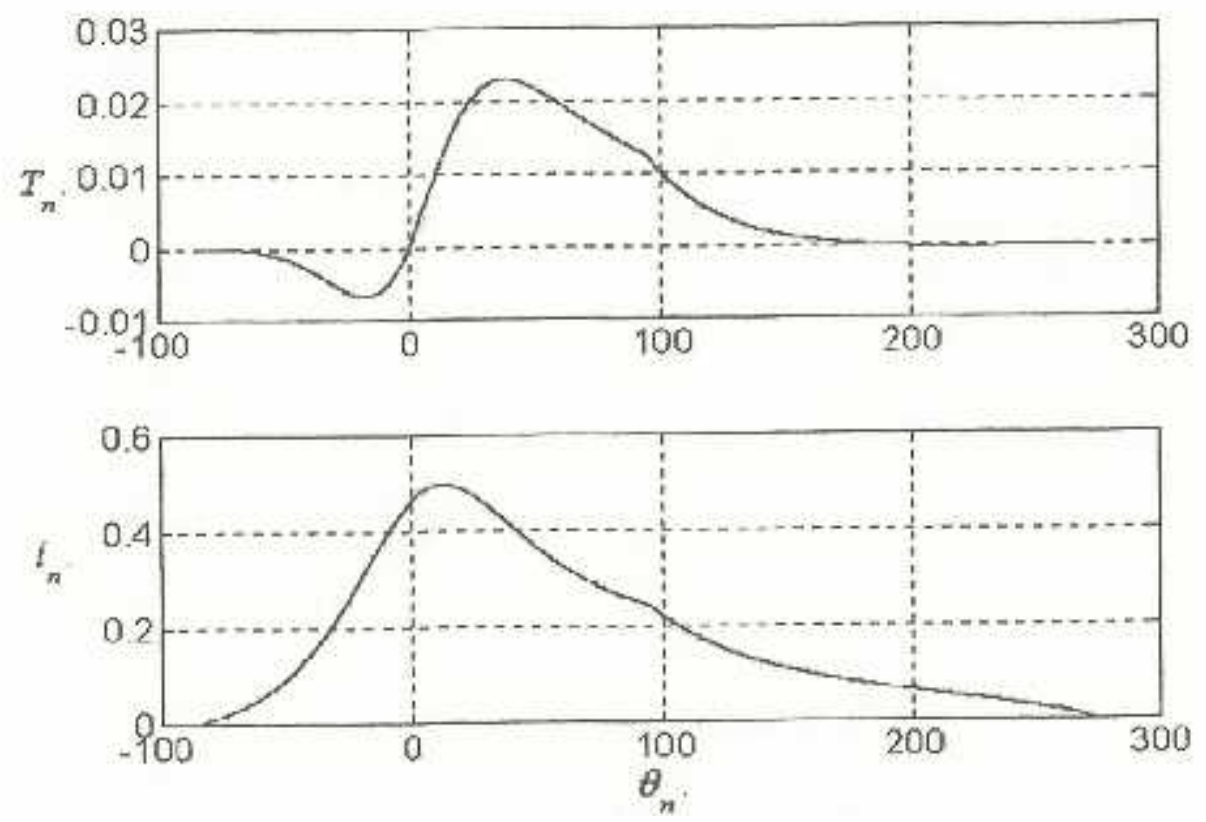

FIG.10- An arbitrary pattern of an individual phase current and the corresponding developed torque, dynamic regime.

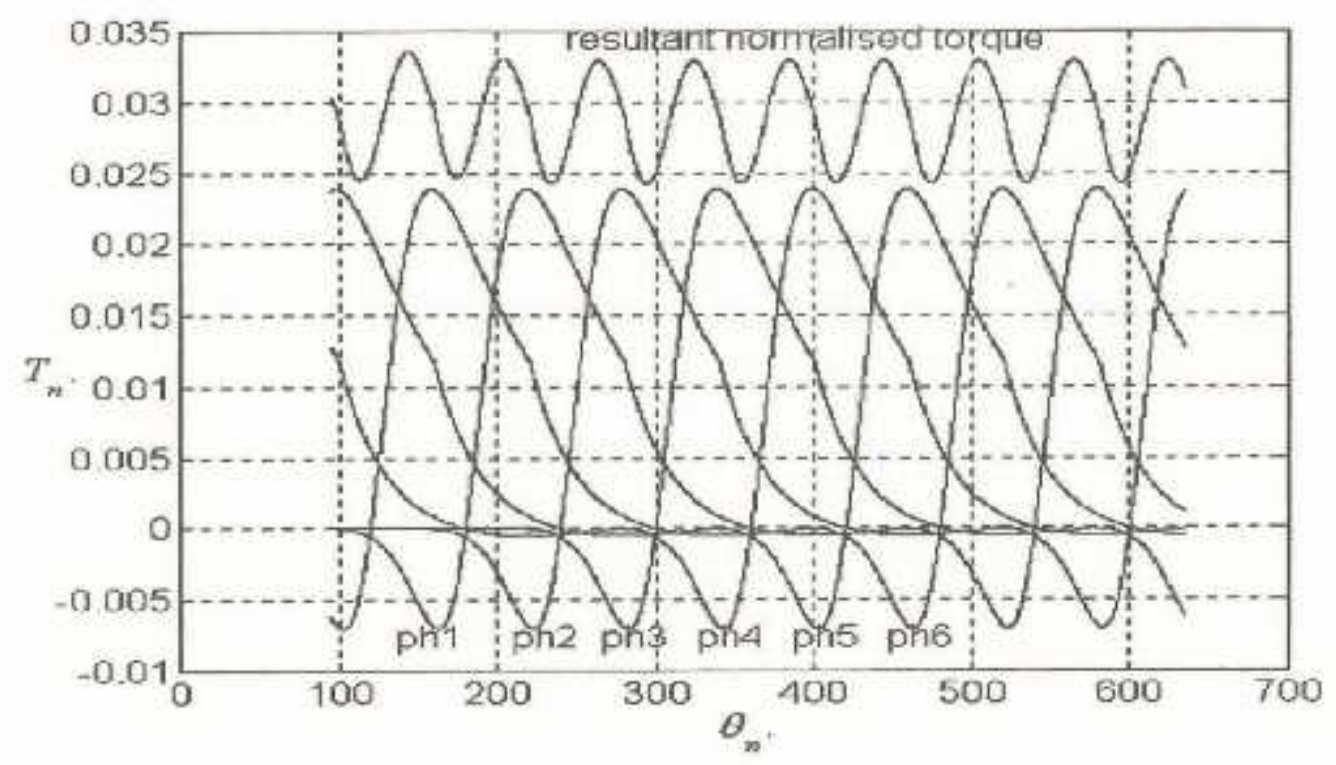

FIG.11- Torque developed by individual phase and the resultant torque of 12/10 motor, dynamic regime 


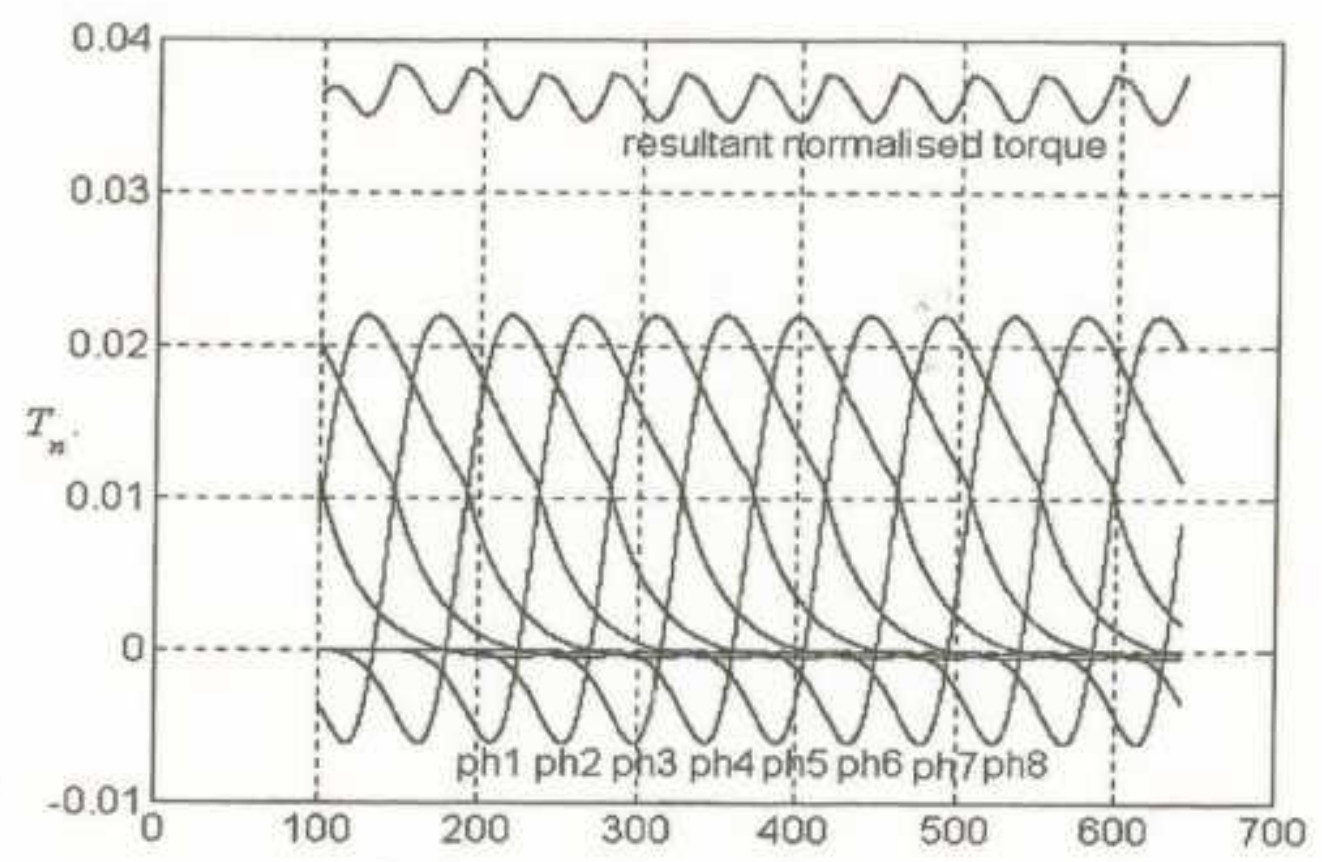

FIG.12- Torque developed by individual phase and the resultant torque of 16/14 motor, dynamic regime

It happens that equation (3) is also very useful to calculate the rate of the torque ripples in dynamic regime. This fact can be checked in FIG.10, FIG.11 and FIG.12. The FIG.10 shows an arbitrary given pattern of a phase current in dynamic regime and the corresponding curve of the torque developed on the shaft for an arbitrary angular velocity. FIG.11 shows the torque developed individually by each phase of a 12/10 motor as well as the resultant dynamic torque in the corresponding regime. Similarly, FIG.12 shows the torque developed individually by each phase of a 16/14 motor as well as the resultant torque applied to the motor's shaft. As can be verified in these figures the rate of the torque ripples in static regime as well as in dynamic regime is around $15 \%$ for the $12 / 10$ motor and around $8 \%$ for the $16 / 14$ motor, as can be calculated from equation (3).

\section{3- Chopper-Controlled DC Commutator-less Motor}

The schematic of a chopper-controlled DC commutator-less motor is shown in FIG.13, which is to be compared to FIG.1. In this figure the DC motor is an interlaced 6-phase 12/10 motor which, as discussed above, can be fed and controlled through a DC/DC converter (chopper) and, in this respect, is recognized as a DC motor. However, this is a DC commutatorless motor in which its mechanical commutator is replaced by an electronic commutator and, in this respect, does not have the drawbacks and limitations of a classical DC commutator motor. 


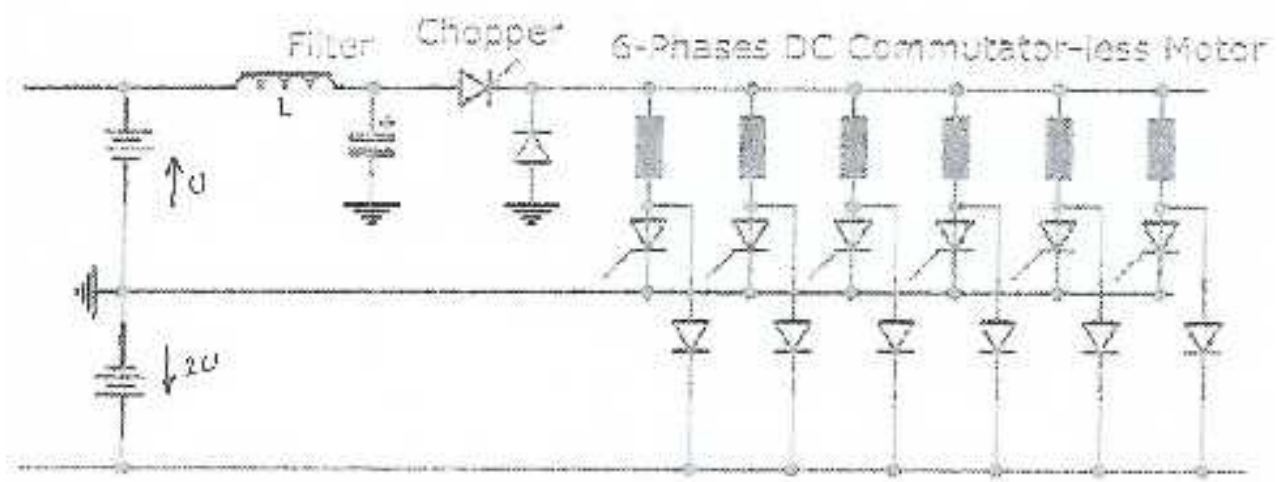

FIG.13- Chopper-Controlled DC 12/10 Commutator-less drive

The chopper-controlled DC commutator-less drive using an appropriate commutator-less motor of standard design $(12 / 10,16 / 14 ; 20 / 18 ; 28 / 26 \ldots)$ can advantageously replace the chopper-controlled DC commutator motor for all high power industrial variable speed applications such as rail traction, ship propulsion, large water pumping, metallurgical industry etc...

Implementation of the drive is quite similar to that of a chopper controlled DC commutator drive. FIG.14 shows the industrial implementation of a chopper controlled 6phase DC commutator-less drive.

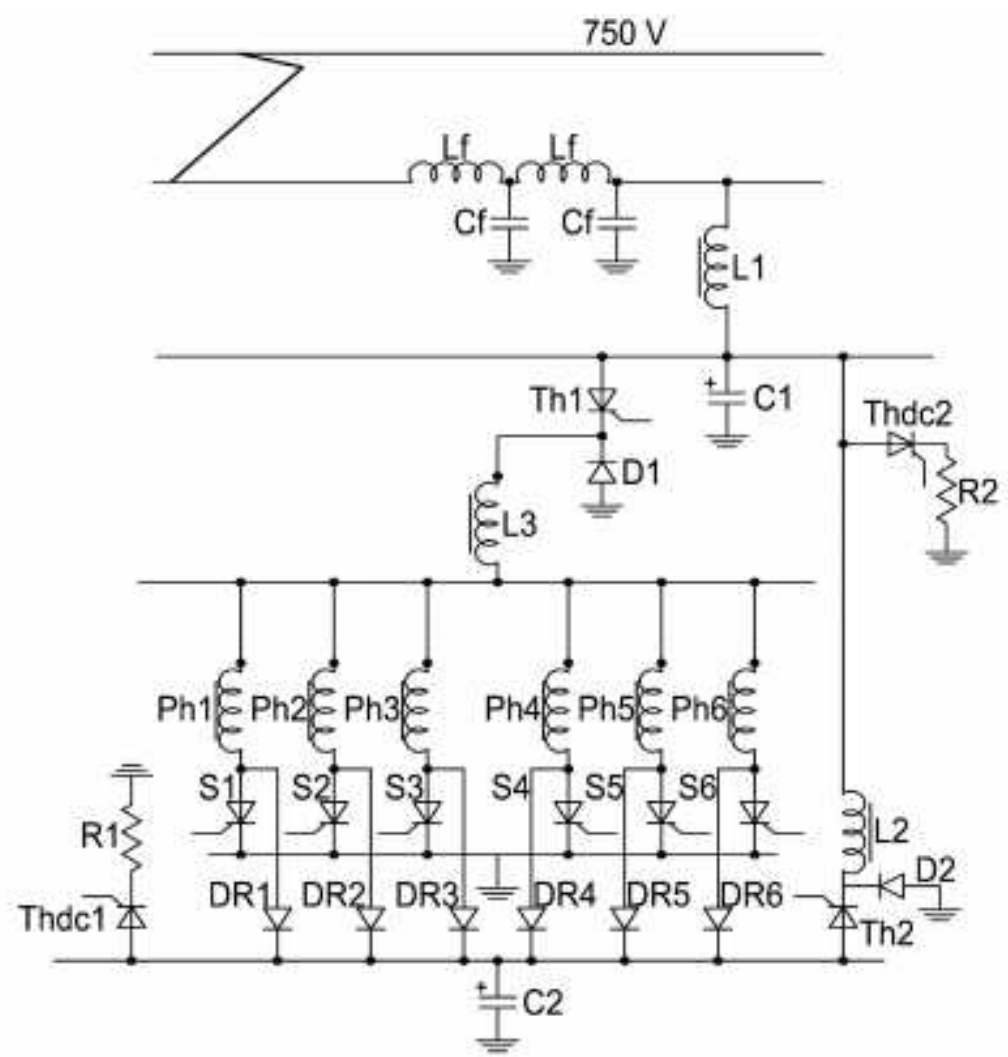

FIG.14- Power alimentation and Control circuitry of a chopper-controlled 6-phase DC commutator-less motor for traction purposes fed through a catenary of $750 \mathrm{~V}$. 
The FIG.14 represents the schematic of the power electronic alimentation of a 6phase, $12 / 10 \mathrm{DC}$ commutator-less traction motor fed through a catenary of $750 \mathrm{~V}$. In this figure we have presented only the main electronic components; the secondary components such as contactors, fast fuses, crow bars, snubbers etc. are not represented. starting from the top, there are two high frequency filters Lf-Cf in series with alimentation line. Next, we encounter the principal filter formed by a large inductor $L_{1}$ and the bank capacitor $C_{1}$. The output of the bank capacitor is linked with the input of a DC/DC converter shown as Th1-D1 in the FIG.14; this is generally an interlaced multiphase chopper, using large, fast thyristors of $300 \mathrm{~Hz}$ frequency and providing a large silicon surface area. (If the available source of energy is a three phase alternative current, one can use a phase-controlled rectifier bridge to attain the same results). At the output of this chopper an adjustable mean DC voltage, UoN, is fabricated. Actually, if the fixed period of the chopper's clock is noted T and the adjustable ON-state time of the chopper's thyristors is ToN, the mean DC voltage at the output of the chopper would be:

$$
U_{O N}=\left(T_{O N} / T\right) U_{0} \quad\left(U_{0}=750 \mathrm{~V}\right)
$$

Hence, if the adjustable ON-state time of the chopper's thyristors ranged from ToN $=\mathrm{T} / 50$ to ToN $=4 T / 5$, the mean DC voltage at the output of the chopper would range from $15 \mathrm{~V}$ to 600 V. Furthermore, if, for example, five interlaced choppers are used, the frequency of the low magnitude current ripples at the chopper's output would be $1500 \mathrm{HZ}$ and can easily be smoothed by the smoothing inductor L3. The output terminal of each phase is linked with the input of an independent controllable power switch. The six power switches, S1 to S6, in FIG.14, are shown as thyristors. However, they could have been IGBTs, GTOs, IGCTs and even power transistors. The output of each controllable power switch is, on one hand, grounded; on the other hand, they are linked, via six recuperative fast diodes DR1 to DR6, to the positive armature of a large damper capacitor $\mathrm{C}_{2}$. Before starting the drive, the dumper capacitor $\mathrm{C}_{2}$ is charged, using the main switches S1 to S6 as step-up choppers, up to Uoff $=1200 \mathrm{~V}$. The voltage of the damper capacitor $\mathrm{C} 2$ is maintained constant equal to $1200 \mathrm{~V}$ during the functioning of the drive, using the step-down chopper, TH2-D2, FIG.14. As stated before, a DC commutatorless motor of standard design having " $2 \mathrm{~s}$ " phases can work in a single-shoot mode with " $\mathrm{s}$ " simultaneously torque productive phases at any instant. Actually, a phase numbered " $\mathrm{n}$ ", controlled by the single power switch $S n$, and the phase numbered " $n+s$ ", controlled by the single power switch $\mathbf{S}_{n+s}$, are, in a standard motor design, conjugate or complementary, in the sense that when $S_{n+s}$ turns ON, $S_{n}$ turns OFF automatically and vice versa. Note that when the power switch Sn turns off, the input terminal of the phase " $n$ " is at the electrical potential UoN and its output terminal at the electrical potential Uoff $>$ > Uon. In this way the current in the phase " $n$ " extinguishes and the magnetic energy of the phase is transferred as electrical energy into the damper bank capacitor $\mathrm{C}_{2}$, causing the increase of voltage between its armatures. However, one can maintain the voltage of the damper capacitor C2 at a constant value (say $1200 \mathrm{~V}$ ) by recovering the excess energy transferred to it using the step down chopper TH2D2 and re-inject this energy to the bank capacitor $\mathrm{C} 1$. In fact, assume that the voltage of C1 is $750 \mathrm{~V}$ and that of $\mathrm{C} 2$ is to be constant equal to $1200 \mathrm{~V}$. Now if the clock period of TH2-D2 is T, the time TON of the chopper TH2-D2 should be set slightly greater than $(750 / 1200)=0.63 T$, that is, $63 \%$ of T. The same step down chopper TH2-D2 is also used for regenerative braking purposes. For this, an arbitrary phase is energized near its minimum reluctance position angle. In this position the two poles of the phase are magnetized; when the minimum reluctance position is reached they oppose further movement of the rotor. As the rotor continues to revolve due to its kinetic energy, the phase current increases in order to more energetically oppose the rotor to move. Finally, when the phase power switch turns OFF all the magnetic energy of the phase is first transferred to the capacitor $\mathrm{C} 2$, then after to the capacitor $\mathrm{C} 1$ using the chopper TH2-D2. This completes the regenerative braking process. 


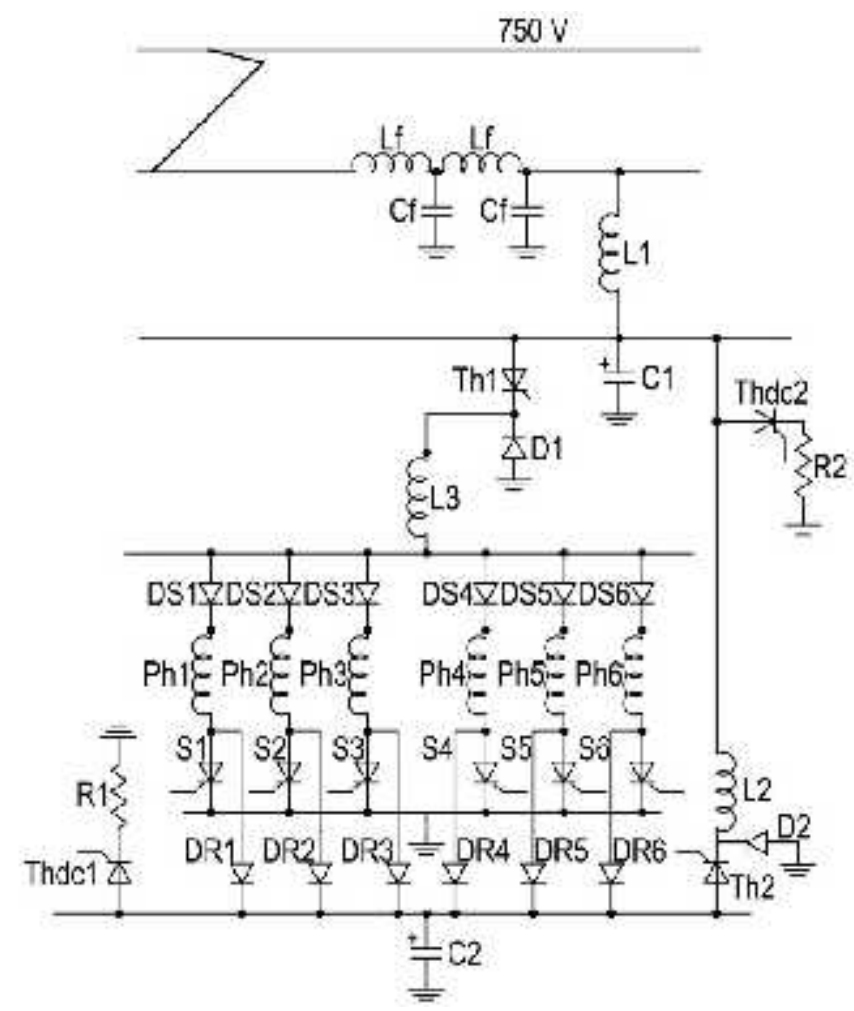

FIG.15- Step 1. Rearrangement of the power electronic feeding circuitry of FIG.14.

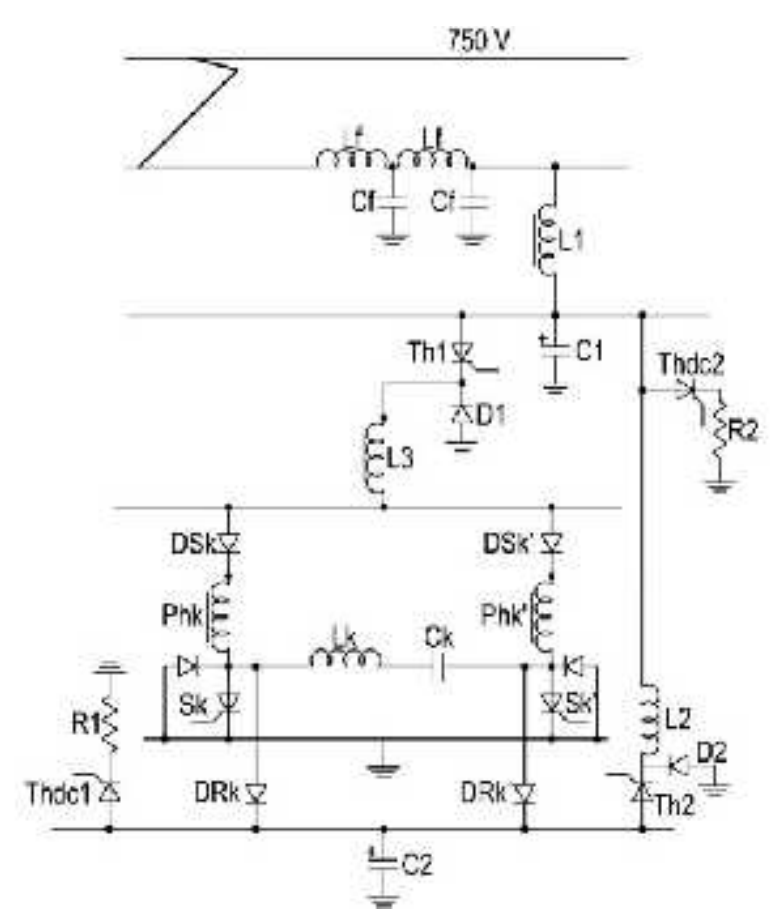

FIG.16- Step 2. Two complementary phases, phk and phk', linked by a series connected LKCK components in order to force the main switches phk and phk' to commutate under zerocurrent 
There exists an interesting and innovative variant of the feeding circuitry of FIG.14 obtained by a rearrangement which we will describe below in two steps using the FIG.15 and the FIG.16. We proceed in two steps in order to not overcharge the final figure obtained after these rearrangements.

The FIG.15 represents the same power electronic circuitry than as shown in FIG.14 except for the presence of six power diodes, DS1 to DS6, placed in series at the input terminals of each phase, $\mathrm{Ph} 1$ to $\mathrm{Ph} 6$, respectively.

The FIG.16 shows two complementary phases, phk and phk', shown in FIG.15. For a 12/10 motor, $k$ and $k^{\prime}$ could takes the following values: $\left(k=1 ; k^{\prime}=4\right) ;\left(k=2 ; k^{\prime}=5\right) ;\left(k=3 ; k^{\prime}=6\right) ;(k=4$; $\left.k^{\prime}=1\right) ;\left(k=5 ; k^{\prime}=2\right) ;\left(k=6 ; k^{\prime}=3\right)$. Let the output terminals of the two conjugate phases Phk and Phk' be linked by the branch Lk-Ck formed by the capacitor Ck and the inductor Lk connected in series as shown in FIG.16. Assume that the power switch Sk is ON and its conjugate $\mathrm{Sk}^{\prime}$ is OFF. The capacitor $\mathrm{Ck}$ will be charged through the phase Phk and its armature connected to the power switch Sk' becomes positive. Then if Sk' is triggered and becomes ON, the capacitor $\mathrm{Ck}$ begins to discharge through the two power switches $\mathrm{Sk}$ and $\mathrm{Sk}^{\prime}$. If the values of $\mathrm{Ck}$ and $\mathrm{Lk}$ are appropriately chosen, first Sk turns OFF and the current continues to flow through the antiparallel diode connected across Sk. If few microseconds after the current through Sk is disappeared a very weak negative voltage is applied to the trigger terminal of the power switch $\mathrm{Sk}$, the latter commute without any tail current and commutation losses. The capacitor $\mathrm{Ck}$ then starts to be charged with the opposite polarity and its voltage increases until it becomes slightly greater than the voltage Uoff of the dumping capacitor C2 (say $1200 \mathrm{~V}$ ), at which instant the recuperative fast diode DRk turns $\mathrm{ON}$ and applies the voltage Uoff to the output terminal of the power switch Sk, in order to transfer the magnetic energy of the Phk phase into the damper capacitor $\mathrm{C2}$. For the next half period, the power switch Sk' turns $\mathrm{ON}$ and the capacitor $\mathrm{Ck}$ is charged in the opposite sense and so on...

Let us choose some numerical values and see how well this power electronic circuit can work and what are the constraints imposed on the power switches. Let $\mathrm{Ck}=25 \mu \mathrm{F}, \mathrm{Lk}=16 \mu \mathrm{H}$ the resonance frequency of the Lk-Ck circuit amounts to:

$\mathrm{f}=\frac{1}{2 \pi \sqrt{L}}=7.96 \mathrm{k}$

The peak current in LK-Ck for the voltage of $1200 \mathrm{~V}$ is

$$
\text { Ipeak }=1200 \sqrt{\frac{\bar{C}}{L}}=1500 \mathrm{~A}
$$

For a peak current $1500 \mathrm{~A}$, the dv/dt imposed over each power switch is

$\mathrm{dv} / \mathrm{dt}=1500 / 25=60 \mathrm{~V} / \mu \mathrm{s}$

For a peak voltage of $1200 \mathrm{~V}$, the di/dt imposed to each power switch is

$\mathrm{di} / \mathrm{dt}=$ Upeak $/ \mathrm{Lk}=1200 / 16=75 \mathrm{~A} / \mu \mathrm{s}$

One must remember that the peak voltage imposed upon each power switch is firmly limited by the large damper capacitor $\mathrm{C} 2$ at the value of $1200 \mathrm{~V}$ in the power electronic circuit we have dealing with. On the other hand, the Lk-Ck branch connected to the input terminals of Sk and $\mathrm{Sk}^{\prime}$ works as an efficient snubber without loss. Actually the energy accumulated in $\mathrm{Ck}$ is used for turning OFF the power switches Sk, and Sk' in each half period.

As we can see, with the numerical values adopted for $\mathrm{Ck}$ and $\mathrm{Lk}$ the constraints imposed on the main power switches are quite weak. Under these conditions, a power IGBT can commute at a few $\mathrm{kHz}$. For example, at $2.5 \mathrm{kHz}$ switching frequency a $12 / 10$ commutatorless motor can be driven at 15,000 RPM and a 16/14 motor at 10,000 RPM. Recall that the 
rotor of a DC commutator-less motor is made only of stacked laminations; it does not use permanent magnets and has not any windings; in this respect, this category of motors can safely revolve at a very high angular velocity and can exhibit quite a high power density (power per unit of mass or volume.) This revels how interesting they can be for traction purposes in electrical vehicles (EV) applications. $\approx$

For illustration purposes, one can propose a 16/14 water-cooled, commutator-less drive fed and controlled by a DC/DC converter having the following characteristics: Power 150 $\mathrm{kW}$; Voltage $600 \mathrm{~V}$ (battery); single air gap length $0.6 \mathrm{~mm}$; maximal speed 18,000 RPM. The switching frequency at 18,000 RPM would be $f_{s}=4,2 \mathrm{kHz}$. It is clear that for working at these high frequencies the phase coils must be carefully fabricated using divided stranded wires in order to limit the supplementary copper losses.

At the other extreme, these drives could be particularly convenient for designing large and low angular speed drives: Imagine a high voltage (U1= $4500 \mathrm{~V}), 4 \mathrm{~s} /(4 \mathrm{~s}-2), 20 \mathrm{MW}, 720$ RPM, 2s-phases, bipolar, chopper-controlled (or phase-controlled rectifier bridge) DC commutator-less drives using a speed-down gearbox of ratio 1/4 for ship propulsion applications (the propeller thus would turn at 180 RPM.) Such a drive would exhibit a rate of torque ripples given by Eq. 3 and would use only $2 \mathrm{~s}$ fast switching thyristors working in the single-shoot mode. If the values of the capacitor $\mathrm{Ck}$ and the inductor Lk are appropriately chosen, thyristors power switches would not need dedicated extinction circuits to turn them OFF. Indeed, in a single shoot mode, each pair of conjugate thyristors, THk and THk', are linked by a Lk-Ck branch. Hence the power switch THk turns OFF automatically when its conjugate THK' is turned ON and, inversely, THk' turns OFF automatically when THk is turned ON. Such a motor would preferentially have a large number of phases to reduce its rate of torque ripples.

As an illustrative example, consider, as a tentative, a 20/18 with $2 \mathrm{~s}=10$ phases DC commutator-less motor working in the single shoot mode for ship propulsion applications. It is assumed that the output shaft of the motor is dotted with a speed-reduction gearbox of ratio $1 / 4$ (the propeller would thus revolve at $1 / 4$ of the speed of the rotor and the torque applied to it would be 4 times greater than that applied to the rotor.) This drive is assumed to have the following characteristics: Bore diameter $D=1800 \mathrm{~mm}$; stack length: $L=500 \mathrm{~mm}$; simple air gap length $\mathrm{g}=4 \mathrm{~mm}$; stator-teeth and rotor-teeth radial height: $\approx 200 \mathrm{~mm}$; width of the stator and rotor-teeth $\approx 115 \mathrm{~mm}$; slot surface area: $\mathrm{Acu} \approx 33500 \mathrm{~mm} 2$; slot filing factor: $\approx 0.35$; primary voltage source: $\mathrm{U}_{1}=4,5 \mathrm{kV}$; secondary voltage source: $\mathrm{U}_{2}=9 \mathrm{kV}$; nominal rotor speed $720 \mathrm{RPM}$; nominal switching frequency in a single-shoot functioning mode $\mathrm{f}_{\mathrm{s}} \approx 216 \mathrm{~Hz}$; nominal rms input voltage per phase Ueff $\approx 3.5 \mathrm{kV}$; nominal rms current per phase: leff $\approx 1.2 \mathrm{kA}$; number of turns per each bipolar phase: $\mathrm{N}=2 \times 25=50$ (in the appendix, section 4 below, a method for calculating the number of turns per phase, $N$, of the excitation winding is presented); nominal rms current density Jeff $\approx 5.0 / \mathrm{mm} 2$; nominal power output $\mathrm{P} \approx 20 \mathrm{MW}$; the nominal power factor as defined by: $\eta=P /(2$ s. Ueff.leff $)$ would be $\approx 0.48$; the rate of the torque ripples would be Tripp $\approx 5 \%$.

It must be underlined that when a single phase is energized, depending on the process of the energization of the phase, a variable amount of reactive magnetizing energy is absorbed by it in order to set its magnetizing flux; and when the same phase is de-energized, this magnetizing energy is recuperated as electrical energy by the bank capacitor C2 (also named the secondary voltage source.) This is why the power factor of each single-phase motor is rather low $(n<0.5)$. However, the recuperated magnetizing energy is not lost; it is reinjected in the successive $\mathrm{ON}$-going phases in order to set their magnetizing flux. The bank capacitor $\mathrm{C} 2$ is thus the source of the reactive power of the drive.

There is also the possibility to design and determine the characteristics of choppercontrolled DC commutator-less drives of more common speed range, for various industrial applications. In the reference [1] at the end of this paper, a realistic example of a choppercontrolled DC commutator-less drive used in railways transportation is fully developed. 


\section{4- Appendix \\ (Calculating the Number of Turns per Phase.)}

The determination of the number of turns of the excitation winding per phase is carried out in several steps through an iterative process, using the transient regime analysis of VRMs as indicated in reference (2). Let us first describe the exact position of the problem and explain clearly its theoretical solution. In a second step we will show how this theoretical solution can be approached numerically:

1- Assume that the geometrical and the dimensional structure of the machine are known.

2- Suppose that in the rated regime the applied phase voltage (constant) is UoN, the rated angular velocity (constant) is $\omega$ and the rated output power is $P$; thus the rated output torque is $T=P / \omega$.

3- Let the number of turns of the excitation winding be $\mathrm{N}$, which is unknown but we are searching to determine its appropriate value.

4- In a single shoot mode functioning, at some initial position angle $\Theta_{0}$ the positive voltage UoN is applied to the phase winding in order to establish an amount of current in that phase. The phase voltage is maintained from $\Theta_{0}$ to the position angle $\Theta_{0}+180^{\circ}$. From the position angle $\Theta 0+180^{\circ}$ to the position angle $\Theta 0+360^{\circ}$ the applied voltage is changed to -Uoff in order to force the phase current to vanish. The phase current would thus vary according to the following general equation:

$\mathrm{u}=K+\frac{d(\theta, i)}{d}$

$\mathrm{u}=K+\frac{o(\theta, i)}{\sigma} \frac{d}{d}+\frac{\delta(\theta, i)}{\delta} \frac{a}{a} \frac{a}{a}$

Denoting $\omega=\frac{d}{d}$, Eq.(A2) is written as

$\left.\mathrm{u}=K+\left[\frac{\delta(\theta, i)}{\partial}\right)+\frac{\delta(\theta, l)}{\theta} \frac{d}{d}\right] \omega$

Finally, the power factor of each single phase motor is defined as

$$
\mathrm{\eta}=\frac{P}{u \quad l}
$$

The Eq.(A3) is a formidable nonlinear differential equation in $t(\theta)$ the coefficients of which, $\delta(\theta, \mathrm{t}) / \delta$ and $\delta(\theta, \mathrm{t}) / \delta$, depend on both $\theta$ and $\mathrm{t}$. In addition, because of the magnetic saturation, the expressions of these two coefficients cannot be easily and accurately determined. However, in the reference [2], one can find a quite easy algorithm to determine them and to solve numerically this differential equation step by step (say every $10^{\circ}$ angle, from $\theta_{0}$ to $\theta_{0}+180^{\circ}$ with the applied phase voltage + Uon and from $\Theta_{0}+180^{\circ}$ to $\theta_{0}+360^{\circ}$ with the applied phase voltage -Uoff). Doing so we will obtain 36 points for the sampled curve of variations of the current $t(\theta)$ and the same number of points for the corresponding sampled curve of torque $T(\theta)$. These two sampled curves resemble to those sketched in FIG.10, from which one can calculate the effective (RMS) phase current and the average output torque as:

$$
\begin{aligned}
& \text { ieff }=\sqrt{\left[\begin{array}{ll}
\sum_{k=1}^{k=3} & \left.\mathrm{t}^{2}(\theta)\right] / 36
\end{array}\right.} \\
& \mathrm{Tav}=\left[\begin{array}{lll}
\sum_{k=1}^{k=3} & \mathrm{~T}(\theta)] / 36
\end{array}\right.
\end{aligned}
$$


Tab.A1 Values of $\Theta_{0}$, ieff and Tav for a given value of $\mathrm{N}$

\begin{tabular}{|l|l|l|l|l|l|l|l|l|}
\hline Өo & $-5^{\circ}$ & $-10^{\circ}$ & $-15^{\circ}$ & $-20^{\circ}$ & $-25^{\circ}$ & $-30^{\circ}$ & $-35^{\circ}$ & etc... \\
\hline ieff & $x x$ & $x x$ & $x x$ & $x x$ & $x x$ & $x x$ & $x x$ & $x x$ \\
\hline Tav & $x x x$ & $x x x$ & $x x x$ & $x x x$ & $x x x$ & $x x x$ & $x x x$ & $x x x$ \\
\hline$\eta$ & $x x x x$ & $x x x x$ & $x x x x$ & $x x x x$ & $x x x x$ & $x x x x$ & $x x x x$ & $x x x x$ \\
\hline
\end{tabular}

Now, one can draw a table with four lines and the required numbers of columns as below: In comparing values of the calculated average torques, Tav, in Tab.A1 to the required value of the torque $\mathrm{T}=\mathrm{P} / \omega$, one can obtain some conclusion concerning the initial chosen value $\mathrm{N}$ of the number of turns o per phase:

1- If all values of Tav calculated in Tab.A1 are less than the required torque $T=P / \omega$, this means that the chosen number of turns $\mathrm{N}$ is too large, and that the required torque cannot be reached with the available voltage $u$ at the angular velocity $\omega$. In that case the above calculations must be resumed with a reduced number of turns $\mathrm{N}^{*}<\mathrm{N}$ and a new table, similar to Tab.A1, must be drawn.

New Tab.A1 giving values of $\Theta 0$, ieff and Tav for a new value of $\mathrm{N}^{*}<\mathrm{N}$

\begin{tabular}{|l|l|l|l|l|l|l|l|l|}
\hline Ө0 & $-5^{\circ}$ & $-10^{\circ}$ & $-15^{\circ}$ & $-20^{\circ}$ & $-25^{\circ}$ & $-30^{\circ}$ & $-35^{\circ}$ & etc... \\
\hline ieff & $\mathrm{xx}$ & $\mathrm{xx}$ & $\mathrm{xx}$ & $\mathrm{xx}$ & $\mathrm{xx}$ & $\mathrm{xx}$ & $\mathrm{xx}$ & $\mathrm{xx}$ \\
\hline Tav & $\mathrm{xxx}$ & $\mathrm{xxx}$ & $\mathrm{xxx}$ & $\mathrm{xxx}$ & $\mathrm{xxx}$ & $\mathrm{xxx}$ & $\mathrm{xxx}$ & $\mathrm{xxx}$ \\
\hline$\eta$ & $\mathrm{xxxx}$ & $\eta 1$ & $\eta 2$ & $\eta 3$ & $\mathrm{xxxx}$ & $\mathrm{xxxx}$ & $\mathrm{xxxx}$ & $\mathrm{xxxx}$ \\
\hline
\end{tabular}

2- In the new table, with $\mathrm{N}^{*}<\mathrm{N}$, it is suggested that for several values of $\Theta_{0}$ the required torque can be reached; however, each acceptable choice of $\Theta$ o will lead to a different value of power factor. One can choose $\mathrm{N}^{*}$ for the number of turns of the phase winding and adopt for the initial firing angle $\Theta_{0}$ the one which gives the highest power factor.

\section{5- Conclusion}

In this paper we have presented a new category of variable speed drive called "chopper-controlled (or "phase controlled rectifier bridge" for an available AC primary source of electrical energy) DC commutator-less drive" whose functioning is exactly similar to the well-known, classical "chopper-controlled DC commutator drives" used in industry over five decades (since 1970 's) as variable speed drives in general, and for electrical traction and propulsion purposes in particular. Both systems use a DC/DC converter (chopper) for feeding and controlling a DC motor. However, the chopper-controlled DC commutator drive has the major drawback of using a commutator DC motor, that is, a classical DC motor having a fragile and expensive mechanical commutator which requires frequent maintenance. In addition, the motor has sliding brushes subject to wear and uses a cumbersome revolving armature winding on its rotor. On the other hand, the chopper controlled DC commutator-less drive uses the same DC/DC converter as in the previous case. However, it uses a DC motor (that is, a motor which can be fed and controlled through a DC source) without a mechanical commutator, without sliding brushes and without any winding on its rotor. It is clear that the innovative drive we present here for the first time, without any references to it in the technical literatures, is far more high performance that the classical "chopper controlled DC commutator drive. A design example of chopper controlled DC commutator-less drive for traction purpose is given in the reference [1] below. It concerns an alternative to the existing inverter-fed asynchronous traction drive used in Paris Metropolitan Railways having the same following characteristics: Power: 250 kW; Voltage (third rail) 750 V; rated speed 2400 RPM; maximum speed 4200 RPM. 


\section{6- References}

[1] Bahram Amin, Système d'entrainement électrique alimenté à partir d'une source de tension continue, et procédé de commande associé, moteur et équipement d'alimentation électrique mis en œuvre dans ce système. French Patent, publication no : FR3092710, www.inpi.fr, 14/08/2020

[2] B. Amin \& All, Field and Torque Calculation and Transient Analysis in Variable Reluctance Machines, IEEE Transactions on Magnetics, 53 (9), PP.1-9, 2017. 